\title{
Indian TB cases highlight need for drug-resistance tests
}

In the last decade, global health experts have sounded the alarms about multidrug-resistant tuberculosis and its even more frightening cousin, extensively drug-resistant tuberculosis. But over the past few months, news from India about a dozen cases of so-called totally drug-resistant (TDR) tuberculosis has led to a renewed call for cheap tests that can rapidly identify resistant forms of the disease. "Totally resistant tuberculosis is out there, make no mistake," says Mel Spigelman, chief executive of the TB Alliance in New York. "The reports from India shouldn't come as a surprise to anybody, but it should come as a wake-up call for the world. We need better diagnostics, surveillance and treatment right away."

Beyond India, TDR tuberculosis has cropped up in Italy and Iran. Meanwhile, a February paper authored by experts from the Stop TB Department at the World Health Organization (WHO) in Geneva estimated that the rate of multidrug-resistant tuberculosis has exceeded $50 \%$ in some countries, such as Moldova and Belarus (Bull. World Health Organ. 90, 111$119,2012)$. The report links this high rate of resistance in part to the overuse of tuberculosis medications in people infected with the lung disease who have not been tested for drugresistant forms of the pathogen.

Around the world, the gold standard to test for drug resistance is still the glacially slow and labor-intensive process of taking sputum samples and culturing them to see whether they are susceptible to each drug. Because tuberculosis bacteria grow slowly, these tests take at least six to eight weeks before the results make it back to the physician, explains William Bishai, co-director of the Johns Hopkins Center for Tuberculosis Research in Baltimore. "In the
US and first-world countries, culture-based drug susceptibility testing is the standard of care and is widely used," he says. But "in resourcepoor countries, [such testing] is not widely available. Clinicians suspect drug resistance only when patients fail to get better after many months of standard therapy."

Two years ago, the WHO endorsed an advanced molecular diagnostic test for tuberculosis drug susceptibility in countries with a high prevalence of the resistant form of the disease. The system, called GeneXpert, analyzes DNA for point mutations that confer resistance to the first-line tuberculosis drug rifampicin. It uses disposable cartridges, can be housed on site and requires little training to run-all big advances over sputum culture tests. As part if its endorsement of GeneXpert, the WHO also worked out a deal with the test manufacturer, Sunnyvale, California's Cepheid, whereby lower income countries such as India could purchase the system at a reduced price pending increased market volume.

Still, the relatively high cost of the GeneXpert test-currently more than $\$ 15$ per personhas led to sluggish adoption in countries with high rates of drug resistance. For example, fewer than two dozen machines have been sold to hospitals in India so far, according to the WHO. And epidemiologists, physicians and researchers all agree that the test falls far short of what is needed, despite its many benefits over culture tests. GeneXpert tests only for resistance to one of the five first-line drugs and five additional second-line drugs for tuberculosis, which means, "unfortunately, GeneXpert still needs to be backed up with a conventional [sputum culture] reference lab," says Karin Weyer, a program coordinator for the WHO Stop TB Department. A cheap and reliable test for resistance to all of the first- and second-line drugs, not just one or two, would allow physicians to make better decisions about which treatment to give initially.

\section{A sensitivity issue}

More comprehensive diagnostic systems are under development by Cepheid and its competitors, among them Belgium's Innogenetics and Germany's Hain Lifescience, but there are still fundamental challenges to overcome, says physician Ruth McNerney of the London School of Hygiene and Tropical Medicine. "Rifampicin we know the mutations for," she explains. But for other tuberculosis drugs such as isoniazid, "the tests we have are less sensitive because there are lots of places it can cause mutations."

To circumvent such barriers, companies have been focusing resources on the development of line probe assays, which use colored bands similar to those in a home pregnancy test to visualize the presence of the bacterium's DNA. The latest line probe assay systems developed by Hain and Innogenetics are capable of detecting resistance to both first- and secondline drugs.

McNerney warns, however, that although line-probe assays are an advance over sputum culture, "they are technically challenging and contamination prone," and so they are not a good solution for much of the world. Instead, she says, money and effort should be focused on the difficult work of creating a better catalog of point-mutation targets, the first step toward developing a cheap, safe and instant DNA-based resistance test.

Rebecca Hersher
Gennova Vaccine Formulation Center, with the remaining money pledged by Gennova. The Indian drugmaker will also provide the expertise to support the facility's construction and operation. The IDRI brings its pipeline of adjuvants to the table.

"I am really very excited about this partnership and the prospects of formulating vaccines in India that will be affordable," MVI's director of research and development Ashley Birkett told Nature Medicine.

The new center builds upon a collaboration launched last year between MVI, Gennova and Tulane University in
New Orleans to accelerate development of a vaccine that interferes with the malaria parasite's replication. But the new center won't be limited to just one disease. "The vaccine candidates for malaria under development at Gennova and by other partners under the MVIPATH umbrella will get the priority, but we will also be able to work for other infectious diseases like leishmaniasis, tuberculosis and human papilloma virus," says Sanjay Singh, chief executive of Gennova.

Still, the first product to undergo testing at the new center will be a malaria vaccine that includes antigens specific to the parasite's sexual stage together with one of three adjuvants that have already been used in human trials in the US and Europe. "If any new adjuvant is found to be more promising," he says, "we will follow guidance from the Indian Department of Biotechnology and the Drugs Controller of India about how to test it in preclinical and clinical studies."

Maharaj Kishan Bhan, secretary for the Indian Department of Biotechnology, applauds the effort. "We welcome this initiative between a private company and nonprofit partners, each with its own strengths," he says.

Killugudi Jayaraman 